\title{
Consolidated Health Economic Evaluation Reporting Standards (CHEERS) Statement
}

\author{
Don Husereau • Michael Drummond - Stavros Petrou • Chris Carswell • \\ David Moher • Dan Greenberg • Federico Augustovski - Andrew H. Briggs • \\ Josephine Mauskopf $\cdot$ Elizabeth Loder
}

Published online: 26 March 2013

(C) Springer International Publishing Switzerland 2013

\begin{abstract}
Economic evaluations of health interventions pose a particular challenge for reporting. There is also a need to consolidate and update existing guidelines and promote their use in a user friendly manner. The Consolidated Health Economic Evaluation Reporting Standards (CHEERS) statement is an attempt to consolidate and update previous health economic evaluation guidelines efforts into one current, useful reporting guidance. The primary audiences for the CHEERS statement are researchers reporting economic evaluations and the editors and peer reviewers assessing them for publication.

The need for new reporting guidance was identified by a survey of medical editors. A list of possible items based on a systematic review was created. A two round, modified Delphi panel consisting of representatives from academia, clinical
\end{abstract}

On behalf of the CHEERS Task Force.

This article is a joint publication by PharmacoEconomics, BJOG: An International Journal of Obstetrics and Gynaecology, BMC Medicine, BMJ, Clinical Therapeutics, Cost Effectiveness and Resource Allocation, The European Journal of Health Economics, International Journal of Technology Assessment in Health Care, Journal of Medical Economics, and Value in Health. Each publisher holds its own copyright.

D. Husereau

Senior Associate

Institute of Health Economics, Edmonton, Canada

D. Husereau

Adjunct Professor of Medicine

Department of Epidemiology and Community Medicine,

University of Ottawa, Ottawa, Canada

D. Husereau

Senior Scientist

University for Health Sciences, Medical Informatics

and Technology, Hall in Tirol, Austria practice, industry, government, and the editorial community was conducted. Out of 44 candidate items, 24 items and accompanying recommendations were developed. The recommendations are contained in a user friendly, 24 item checklist. A copy of the statement, accompanying checklist, and this report can be found on the ISPOR Health Economic Evaluations Publication Guidelines Task Force website (www.ispor.org/TaskForces/EconomicPubGuidelines.asp).

We hope CHEERS will lead to better reporting, and ultimately, better health decisions. To facilitate dissemination and uptake, the CHEERS statement is being copublished across 10 health economics and medical journals. We encourage other journals and groups, to endorse CHEERS. The author team plans to review the checklist for an update in five years.

Health economic evaluations are conducted to inform resource allocation decisions. Economic evaluation has been defined as "the comparative analysis of alternative courses of action in terms of both their costs and their consequences." [1] All economic evaluations assess costs, but approaches to measuring and valuing the consequences of health interventions may differ (see Box).

D. Husereau $(\square)$

879 Winnington Ave, Ottawa, ON K2B 5C4, Canada

e-mail: donh@donhusereau.com

M. Drummond

Professor of Health Economics

Centre for Health Economics, University of York, York, UK

S. Petrou

Professor of Health Economics

Warwick Medical School, University of Warwick,

Coventry, UK 


\section{Forms of economic evaluation [1]}

Specific forms of analysis reflect different approaches to evaluating the consequences of health interventions. Health consequences may be estimated from a single analytical (experimental or non-experimental) study, a synthesis of studies, mathematical modelling, or a combination of modelling and study information.

Cost consequences analysis examines costs and consequences without attempting to isolate a single consequence or aggregate consequences into a single measure.

Cost minimisation analysis (CMA)-The consequences of compared interventions are required to be equivalent, and only relative costs are compared.

Cost effectiveness analysis (CEA) measures consequences in natural units, such as life years gained, disability days avoided, or cases detected. In a variant of CEA, often called cost utility analysis, consequences are measured in terms of preference-based measures of health, such as quality adjusted life years or disability adjusted life years.

Cost benefit analysis-Consequences are valued in monetary units.

Readers should be aware that an economic evaluation might be referred to as a "cost effectiveness analysis" or "cost benefit analysis" even if it does not strictly adhere to the definitions above. Multiple forms may also exist within a single evaluation. Different forms of analysis provide unique advantages or disadvantages for decision making. The Consolidated Health Economic Evaluation Reporting Standards (CHEERS) statement can be used with any form of economic evaluation.

\section{Carswell}

Editor

PharmacoEconomics,

Adis International, Auckland, New Zealand

D. Moher

Senior Scientist

Clinical Epidemiology Program, Ottawa Hospital Research

Institute, Ottawa, Canada

\section{Greenberg}

Associate Professor and Chairman

Department of Health Systems Management,

Faculty of Health Sciences, Ben-Gurion University of the Negev,

Beer-Sheva, Israel

\section{Greenberg}

Visiting Assistant Professor

Center for the Evaluation of Value and Risk in Health,

Tufts Medical Center, Boston, MA, USA
Economic evaluations have been widely applied in health policy, including the assessment of prevention programmes (such as vaccination, screening, and health promotion), diagnostics, treatment interventions (such as drugs and surgical procedures), organisation of care, and rehabilitation. Economic evaluations are increasingly being used for decision making and are an important component of programmes for health technology assessment internationally [2].

\section{Reporting Challenges and Shortcomings in Health Economic Evaluations}

Compared with clinical studies, which report the consequences of an intervention only, economic evaluations require more reporting space for additional items, such as resource use, costs, preference related information, and cost effectiveness results. This creates challenges for editors, reviewers, and those who wish to scrutinise a study's findings [3]. There is evidence that the quality of reporting of economic evaluations varies widely and could potentially benefit from improved quality assurance mechanisms $[4,5]$.

With the increasing number of publications available, and opportunity costs from decisions based on misleading study findings, transparency and clarity in reporting are important. In addition, outside of economic evaluations conducted alongside clinical trials, there are no widespread mechanisms for warehousing economic evaluation data to allow for independent interrogation, such as ethics review proceedings, regulator dossiers, or study registries. Instead, independent analysis may rely on the record keeping of individual investigators.

Even if measures to promote transparency exist, such as registries, biomedical journal editors have increasingly

\section{F. Augustovski}

Director

Health Economic Evaluation and Technology Assessment, Institute for Clinical Effectiveness and Health Policy (IECS), Buenos Aires, Argentina

F. Augustovski

Professor of Public Health

Universidad de Buenos Aires, Buenos Aires, Argentina

\section{A. H. Briggs}

William R. Lindsay Chair of Health Economics

Health Economics and Health Technology Assessment, Institute of Health and Wellbeing, University of Glasgow, Glasgow, Scotland

\author{
J. Mauskopf \\ Vice President of Health Economics \\ RTI Health Solutions, Research Triangle Park, NC, USA
}


promoted and endorsed the use of reporting guidelines. Endorsement of guidelines by journals for randomised controlled trials has been shown to improve reporting [6]. The combination of the risk of making costly decisions due to poor reporting with the lack of mechanisms that promote accountability makes transparency in reporting economic evaluations especially important and a primary concern among journal editors and decision makers $[3,7]$.

\section{Aim and Scope}

The aim of the Consolidated Health Economic Evaluation Reporting Standards (CHEERS) statement is to provide recommendations, in the form of a checklist, to optimise reporting of health economic evaluations. The need for a contemporary reporting guidance for economic evaluations was recently identified by researchers and biomedical journal editors [8]. The CHEERS statement attempts to consolidate and update previous efforts $[9,10,11,12,13,14,15,16,17$, $18,19,20]$ into a single useful reporting guidance.

The primary audiences for the CHEERS statement are researchers reporting economic evaluations and the editors and peer reviewers evaluating their publication potential. We hope the statement (which consists of a 24 item checklist and accompanying recommendations on the minimum amount of information to be included when reporting economic evaluations) is a useful and practical tool for these audiences and will improve reporting and, in turn, health and healthcare decisions. To best understand and apply the recommendations contained within the statement, we encourage readers to access the Explanation and Elaboration Report [21].

\section{Development of the CHEERS Statement}

The statement was developed by a task force supported by the International Society for Pharmacoeconomics and Outcomes Research (ISPOR), as part of a broader initiative to facilitate and encourage the interchange of expert knowledge and develop best practices. The CHEERS Task Force members were chosen by the chair of the task force primarily based on their longstanding academic expertise and contribution to the multidisciplinary field of health

\section{E. Loder}

Clinical Epidemiology Editor

BMJ, London, UK

E. Loder

Chief of Division

Division of Headache and Pain, Brigham and Women's/Faulkner

Neurology, Faulkner Hospital, Boston, MA, USA economic evaluation. In addition to four members of the task force with doctorates in economics and its sub-discipline of health economics (AHB, MD, JM, SP), members included experts in health technology assessment and decision making (FA, AHB, DH, MD, JM) and in clinical epidemiology and biostatistics (AHB, EL, DM), those in active clinical practice (EL, FA), and those with previous experience in reporting guideline development (MD, DM). All members are researchers in applied health and health policy, with five members currently serving as editors for journals in the field (AHB, CC, MD, DG, EL).

The CHEERS Task Force followed current recommendations for developing reporting guidelines [22]. Briefly, the need for new guidance was first identified through a survey of members of the World Association of Medical Editors. Of the 6\% (55/965) who responded, 91\% (n=50) indicated they would use a standard if one were widely available [8]. Next, published checklists or guidance documents related to reporting economic evaluations were identified from a systematic review and survey of task force members [23]. Both of these activities were used to create a preliminary list of items to include when reporting economic evaluations. Recommendations of the minimum set of reporting items were then developed through a modified Delphi panel process. Forty eight individuals identified by the task force with broad geographical representation and representing academia, biomedical journal editors, the pharmaceutical industry, government decision makers, and those in clinical practice were invited to participate. Thirty-seven agreed to participate. Participants were asked to score importance on a Likert scale and the average scores, weighted by each individual's confidence in ability to score, were then used to rank items. A cut-off point was applied to the ranked list to determine the minimum number of items important for reporting.

The CHEERS statement recommendations have been independently reviewed and subsequently revised by task force members. The recommendations are entirely those of the task force-the sponsors of the study had no role in study design, data analysis, data interpretation, or writing of the final recommendations. A more complete description of the methods and findings of the Delphi panel are found in the larger explanation and elaboration document [21].

\section{Checklist Items}

The final recommendations are subdivided into six main categories: (1) title and abstract; (2) introduction; (3) methods; (4) results; (5) discussion; and (6) other. The recommendations are contained in a user friendly, 24 item checklist (Table 1) to aid users who wish to follow them. A copy of the checklist can also be found on the CHEERS 
Table 1 CHEERS checklist-Items to include when reporting economic evaluations of health interventions

\begin{tabular}{|c|c|c|}
\hline Section/item & $\begin{array}{l}\text { Item Recommendation } \\
\text { No }\end{array}$ & $\begin{array}{l}\text { Reported on } \\
\text { page No/ line No }\end{array}$ \\
\hline
\end{tabular}

\section{Title and abstract}

Title

Abstract

\section{Introduction}

Background and objectives

\section{Methods}

Target population and subgroups

Setting and location

Study perspective

Comparators

Time horizon

Discount rate

Choice of health outcomes

Measurement of effectiveness

Measurement and valuation of preference based outcomes

Estimating resources and costs

Currency, price date, and conversion

Choice of model

1
Identify the study as an economic evaluation or use more specific terms such as "cost-effectiveness analysis", and describe the interventions compared.

Provide a structured summary of objectives, perspective, setting, methods (including study design and inputs), results (including base case and uncertainty analyses), and conclusions.

Provide an explicit statement of the broader context for the study.

Present the study question and its relevance for health policy or practice decisions.

Describe characteristics of the base case population and subgroups analysed, including why they were chosen.

State relevant aspects of the system(s) in which the decision(s) need(s) to be made.

Describe the perspective of the study and relate this to the costs being evaluated.

Describe the interventions or strategies being compared and state why they were chosen.

State the time horizon(s) over which costs and consequences are being evaluated and say why appropriate.

Report the choice of discount rate(s) used for costs and outcomes and say why appropriate.

Describe what outcomes were used as the measure(s) of benefit in the evaluation and their relevance for the type of analysis performed.

1a Single study-based estimates: Describe fully the design features of the single effectiveness study and why the single study was a sufficient source of clinical effectiveness data.

12 If applicable, describe the population and methods used to elicit preferences for outcomes.

13a Single study-based economic evaluation: Describe approaches used to estimate resource use associated with the alternative interventions. Describe primary or secondary research methods for valuing each resource item in terms of its unit cost. Describe any adjustments made to approximate to opportunity costs.

13b Model-based economic evaluation: Describe approaches and data sources used to estimate resource use associated with model health states. Describe primary or secondary research methods for valuing each resource item in terms of its unit cost. Describe any adjustments made to approximate to opportunity costs.

14 Report the dates of the estimated resource quantities and unit costs. Describe methods for adjusting estimated unit costs to the year of reported costs if necessary. Describe methods for converting costs into a common currency base and the exchange rate.

Describe and give reasons for the specific type of decision-analytical model used. Providing a figure to show model structure is strongly recommended.

16 Describe all structural or other assumptions underpinning the decisionanalytical model. 
Table 1 continued

\begin{tabular}{|c|c|c|c|}
\hline Section/item & $\begin{array}{l}\text { Item } \\
\text { No }\end{array}$ & Recommendation & $\begin{array}{l}\text { Reported on } \\
\text { page No/ line No }\end{array}$ \\
\hline Analytical methods & 17 & $\begin{array}{l}\text { Describe all analytical methods supporting the evaluation. This could include } \\
\text { methods for dealing with skewed, missing, or censored data; extrapolation } \\
\text { methods; methods for pooling data; approaches to validate or make } \\
\text { adjustments (such as half cycle corrections) to a model; and methods for } \\
\text { handling population heterogeneity and uncertainty. }\end{array}$ & \\
\hline \multicolumn{4}{|l|}{ Results } \\
\hline Study parameters & 18 & $\begin{array}{l}\text { Report the values, ranges, references, and, if used, probability distributions } \\
\text { for all parameters. Report reasons or sources for distributions used to } \\
\text { represent uncertainty where appropriate. Providing a table to show the input } \\
\text { values is strongly recommended. }\end{array}$ & \\
\hline Incremental costs and outcomes & 19 & $\begin{array}{l}\text { For each intervention, report mean values for the main categories of } \\
\text { estimated costs and outcomes of interest, as well as mean differences } \\
\text { between the comparator groups. If applicable, report incremental cost- } \\
\text { effectiveness ratios. }\end{array}$ & \\
\hline \multirow[t]{2}{*}{ Characterising uncertainty } & $20 \mathrm{a}$ & $\begin{array}{l}\text { Single study-based economic evaluation: Describe the effects of sampling } \\
\text { uncertainty for the estimated incremental cost and incremental } \\
\text { effectiveness parameters, together with the impact of methodological } \\
\text { assumptions (such as discount rate, study perspective). }\end{array}$ & \\
\hline & $20 \mathrm{~b}$ & $\begin{array}{l}\text { Model-based economic evaluation: Describe the effects on the results of } \\
\text { uncertainty for all input parameters, and uncertainty related to the structure } \\
\text { of the model and assumptions. }\end{array}$ & \\
\hline Characterising heterogeneity & 21 & $\begin{array}{l}\text { If applicable, report differences in costs, outcomes, or cost-effectiveness that } \\
\text { can be explained by variations between subgroups of patients with different } \\
\text { baseline characteristics or other observed variability in effects that are not } \\
\text { reducible by more information. }\end{array}$ & \\
\hline \multicolumn{4}{|l|}{ Discussion } \\
\hline $\begin{array}{l}\text { Study findings, limitations, } \\
\text { generalisability, and current } \\
\text { knowledge }\end{array}$ & 22 & $\begin{array}{l}\text { Summarise key study findings and describe how they support the conclusions } \\
\text { reached. Discuss limitations and the generalisability of the findings and } \\
\text { how the findings fit with current knowledge. }\end{array}$ & \\
\hline \multicolumn{4}{|l|}{ Other } \\
\hline Source of funding & 23 & $\begin{array}{l}\text { Describe how the study was funded and the role of the funder in the } \\
\text { identification, design, conduct, and reporting of the analysis. Describe other } \\
\text { non-monetary sources of support. }\end{array}$ & \\
\hline Conflicts of interest & 24 & $\begin{array}{l}\text { Describe any potential for conflict of interest of study contributors in } \\
\text { accordance with journal policy. In the absence of a journal policy, we } \\
\text { recommend authors comply with International Committee of Medical } \\
\text { Journal Editors recommendations. }\end{array}$ & \\
\hline
\end{tabular}

For consistency, the CHEERS statement checklist format is based on the format of the CONSORT statement checklist

Task Force website. (www.ispor.org/TaskForces/Economic PubGuidelines.asp). In order to encourage dissemination and use of a single international standard for reporting, the task force approached 14 journals identified as either the largest publishers of economic evaluations or widely read by the medical and research community. Thirteen journals responded, and 10 expressed their ability and interest in endorsing this guidance. The CHEERS statement is being simultaneously published in BMC Medicine, BMJ, BJOG: An International Journal of Obstetrics and Gynaecology, Clinical Therapeutics, Cost Effectiveness and Resource Allocation, The European Journal of Health Economics, International Journal of Technology Assessment in Health
Care, Journal of Medical Economics, PharmacoEconomics, and Value in Health. To facilitate wider dissemination and uptake of this reporting guidance, we encourage other journals and groups to consider endorsing CHEERS.

\section{Concluding Remarks}

As the number of published health economic evaluations continues to grow, we believe more transparent and complete reporting of methods and findings will be increasingly important to facilitate interpretation and comparison of studies. We hope the CHEERS statement, consisting of 
recommendations in a 24 item checklist, will be viewed as an effective consolidation and update of previous efforts and serve as a starting point for standard reporting going forward.

We believe the CHEERS statement represents a considerable expansion over previous efforts. The strength of our approach is that it was developed in accordance with current recommendations for the development of reporting guidelines, using an international and multidisciplinary team of editors and content experts in economic evaluation and reporting [22]. Similar to the approach taken with other widely accepted guidelines, we have defined a minimum set of criteria though a modified Delphi technique and have translated these into recommendations, an explanatory document with explanations, and a checklist. Unlike some previous reporting guidance for economic evaluation, we have also made every effort to be neutral about the conduct of economic evaluation, allowing analysts the freedom to choose different methods.

There may be several limitations to our approach. A larger Delphi panel with a different composition could have led to a different final set of recommendations [24]. Some less common approaches and contexts (such as public health, developing countries, and system dynamic models) for conducting health economic evaluation may not be well represented by our sample of experts. Additionally, like many Delphi panel processes, we based decisions to reject or accept criteria on arbitrary levels of importance. However, we feel the group recruited to create the statement is sufficiently knowledgeable of the more common applications of economic evaluation, and the rules used to select criteria were created a priori and are consistent with previous efforts.

We believe it will be important to evaluate the effects of implementation of this statement and checklist on reporting in future economic evaluations. As methods for the conduct of economic evaluation continue to evolve, it will also be important to revisit or extend the guidance. The CHEERS Task Force feels that this statement should be reviewed for updating five years from its release.

Acknowledgements The International Society for Pharmacoeconomics and Outcomes Research (ISPOR) Health Economic Evaluation Publication Guidelines-CHEERS Task Force acknowledge the support of Elizabeth Molsen; Donna Rindress, who provided the initial leadership for this effort; and the reviewers and Delphi panel participants, who are named in the larger explanation and elaboration document and on the CHEERS Task Force website.

Contributors All authors provided a substantial contribution to the design and interpretation of the protocol and guidance, as well as writing sections of drafts, revising based on comments received, and approving the final version. DH conducted the analysis of Delphi panel responses, drafted and revised the protocol and the drafts of this paper, and is the guarantor for the study.
Competing Interests All authors have completed the ICMJE uniform disclosure form at www.icmje.org/coi_disclosure.pdf and declare: FA served as board member for the study funder; FA, AHB, CC, MD, DG, DH, EL, JM, and SP were provided support for travel to a face-to-face meeting to discuss the contents of the report; FA and MD have received payment from the study sponsor for serving as coeditors for Value in Health; no other relationships or activities that could appear to have influenced the submitted work have occurred. Elizabeth Loder-BMJ clinical epidemiology editor-played no part in the peer review or decision making of this paper at the editorial level, and contributed solely as an author.

Funding All CHEERS Task Force members are volunteers. Support for this initiative was provided by the International Society for Pharmacoeconomics and Outcomes Research.

\section{References}

1. Drummond MF, Sculpher MJ, Torrance G, O’Brien J, Stoddart GL. Methods for the economic evaluation of health care programmes. 3rd ed. New York: Oxford University Press; 2005.

2. Drummond MF, Schwartz JS, Jönsson B, Luce BR, Neumann PJ, Siebert U, et al. Key principles for the improved conduct of health technology assessments for resource allocation decisions. Int J Technol Assess Health Care. 2008;24:244-58.

3. Rennie D, Luft HS. Pharmacoeconomic analyses. JAMA. 2000; 283:2158-60.

4. Neumann PJ, Stone PW, Chapman RH, Sandberg EA, Bell CM. The quality of reporting in published cost-utility analyses, 1976-1997. Ann Intern Med. 2000;132:964.

5. Rosen AB, Greenberg D, Stone PW, Olchanski NV, Neumann PJ. Quality of abstracts of papers reporting original cost-effectiveness analyses. Med Decis Mak. 2005;25:424-8.

6. Turner L, Shamseer L, Altman DG, Schulz KF, Moher D. Does use of the CONSORT Statement impact the completeness of reporting of randomised controlled trials published in medical journals? A Cochrane review. Syst Rev. 2012;1:60.

7. Drummond MF. A reappraisal of economic evaluation of pharmaceuticals. Science or marketing? Pharmacoeconomics. 1998; 14:1-9.

8. McGhan WF, Al M, Doshi JA, Kamae I, Marx SE, Rindress D. The ISPOR good practices for quality improvement of costeffectiveness research task force report. Value Health. 2009;12: 1086-99.

9. Task Force on Principles for Economic Analysis of Health Care Technology. Economic analysis of health care technology. A report on principles. Ann Intern Med. 1995;123:61-70.

10. Drummond MF, Jefferson TO. Guidelines for authors and peer reviewers of economic submissions to the BMJ. BMJ. 1996;313: 275-83.

11. Gold MR. Cost-effectiveness in health and medicine. New York: Oxford University Press; 1996.

12. Siegel JE, Weinstein MC, Russell LB, Gold MR. Recommendations for reporting cost-effectiveness analyses. Panel on CostEffectiveness in Health and Medicine. JAMA. 1996;276: $1339-41$.

13. Nuijten MJ, Pronk MH, Brorens MJ, Hekster YA, Lockefeer JH, de Smet PA, et al. Reporting format for economic evaluation: Part II: focus on modelling studies. Pharmacoeconomics. 1998; 14:259-68.

14. Vintzileos AM, Beazoglou T. Design, execution, interpretation, and reporting of economic evaluation studies in obstetrics. Am J Obstet Gynecol. 2004;191:1070-6. 
15. Drummond M, Manca A, Sculpher M. Increasing the generalizability of economic evaluations: recommendations for the design, analysis, and reporting of studies. Int $\mathrm{J}$ Technol Assess Health Care. 2005;21:165-71.

16. Ramsey S, Willke R, Briggs A, Brown R, Buxton M, Chawla A, et al. Good research practices for cost-effectiveness analysis alongside clinical trials: the ISPOR RCT-CEA Task Force report. Value Health. 2005;8:521-33.

17. Goetghebeur MM, Wagner M, Khoury H, Levitt RJ, Erickson LJ, Rindress D. Evidence and value: impact on decisionmaking- the EVIDEM framework and potential applications. BMC Health Serv Rev. 2008;8:270.

18. Davis JC, Robertson MC, Comans T, Scuffham PA. Guidelines for conducting and reporting economic evaluation of fall prevention strategies. Osteoporos Int. 2010;22:2449-59.

19. Petrou S, Gray A. Economic evaluation using decision analytical modelling: design, conduct, analysis, and reporting. BMJ. 2011; 342:d1766.
20. Petrou S, Gray A. Economic evaluation alongside randomised controlled trials: design, conduct, analysis, and reporting. BMJ. 2011;342:d1548.

21. Husereau D, Drummond M, Petrou S, Carswell C, Moher D, Greenberg D, et al. Consolidated Health Economic Evaluation Reporting Standards (CHEERS) - explanation and elaboration: a report of the ISPOR Health Economic Evaluations Publication Guidelines Task Force. Value in Health (forthcoming).

22. Moher D, Schulz KF, Simera I, Altman DG. Guidance for developers of health research reporting guidelines. PLoS Med. 2010;7:e1000217.

23. Moher D, Weeks L, Ocampo M, et al. Describing reporting guidelines for health research: a systematic review. J Clin Epi 2011;64:718-42.

24. Campbell SM, Hann M, Roland MO, Quayle JA, Shekelle PG. The effect of panel membership and feedback on ratings in a tworound Delphi survey: results of a randomized controlled trial. Med Care. 1999;37:964-8. 